Mots. Les langages du politique

$81 \mid 2006$

Suisse, laboratoire politique européen ?

\title{
Conjurer le désordre discursif. Les procédés de « lissage » dans la fabrication du discours institutionnel
}

Claire Oger et Caroline Ollivier-Yaniv

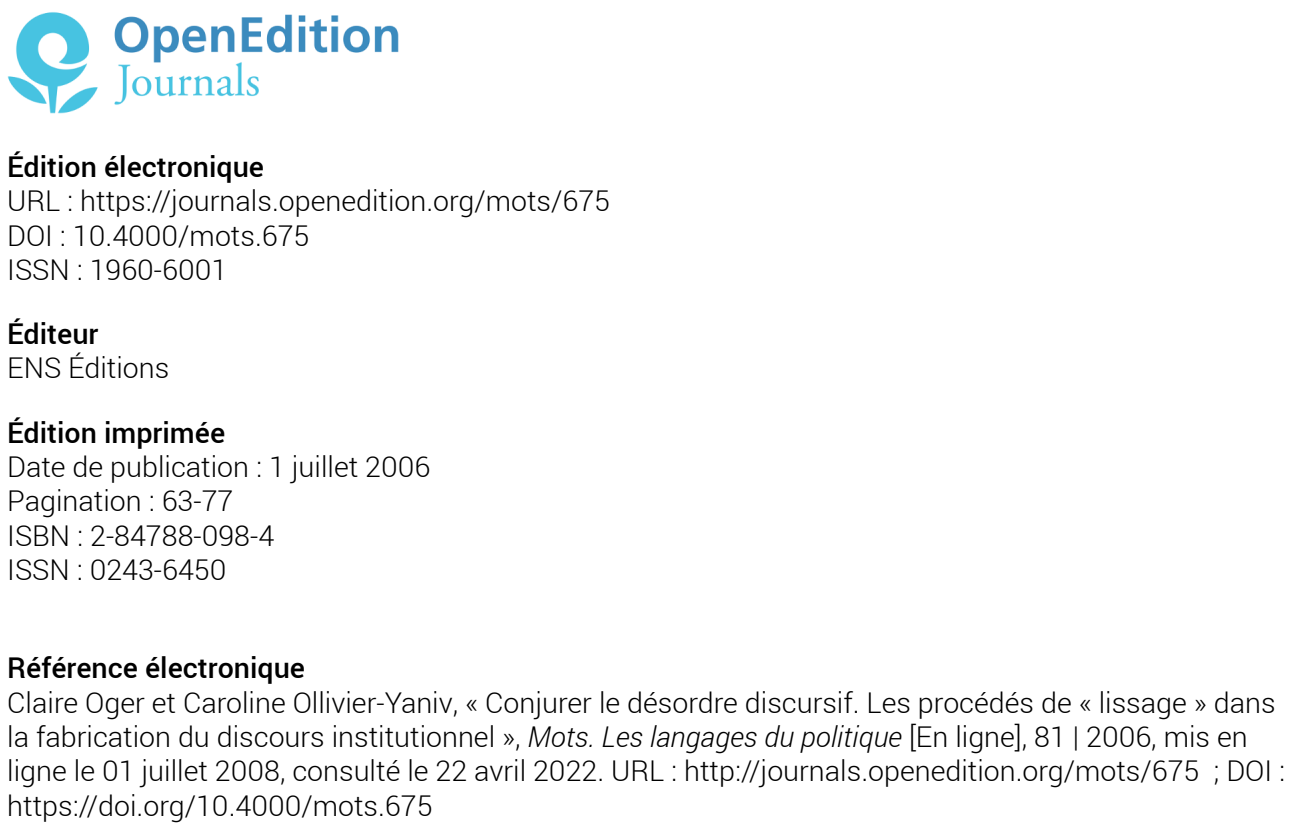

(c) ENS Éditions 


\section{Conjurer le désordre discursif. Les procédés de « lissage » dans la fabrication du discours institutionnel}

À partir de recherches sur la communication des institutions publiques et sur le discours institutionnel (notamment en matière de défense), notre travail commun porte sur l'intérêt de rapprocher l'analyse de discours et la sociologie compréhensive pour étudier le discours institutionnel. Ce croisement des approches nous a conduites à définir une « anthropologie du discours institutionnel ».

Dans un certain nombre de travaux antérieurs ${ }^{1}$, nous avons en effet tenté de mettre en évidence l'existence de plusieurs formes de discours institutionnel correspondant aux différentes échelles de représentation de l'institution. Dans cette perspective, nous avons souhaité distinguer le «discours instituant»sorte d'idéal type du discours officiel de l'institution, pris à tort pour la totalité du discours institutionnel - et les discours institutionnels dont la pluralité renvoie à des situations d'énonciation variables relativement à l'institution (et comportant notamment des positions «semi-officielles»).

Nous abordons ici le discours instituant qu'on trouve dans différents textes de cadrage de la communication institutionnelle d'institutions publiques (et de ministères, en particulier), ainsi que dans les messages véhiculés par certaines campagnes de communication institutionnelle.

La communication institutionnelle doit être comprise ici dans son sens le plus large, c'est-à-dire non limitée aux campagnes de type publicitaire. En particulier pour ce qui concerne la Défense - notre «laboratoire» institutionnel originel -, parler de communication institutionnelle renvoie à des campagnes

1. Les fondements théoriques du rapprochement entre analyse de discours et sociologie compréhensive ont été évoqués dans un article paru en 2003 dans la revue Mots. Les langages du politique (Oger et Ollivier-Yaniv, 2003a), tandis que leurs prolongements méthodologiques (et notamment la nécessité de constituer des corpus hétérogènes pour fonder une « anthropologie des discours institutionnels ») ont été évoqués lors d’une communication à la conférence de Bucarest en 2003 (Oger et Ollivier-Yaniv, 2003b). On trouvera un exemple de mise en œuvre de cette méthode dans un article de C. Oger consacré aux directives de communication de la Défense et publié dans Quaderni (Oger, 2003). 
mais aussi à la communication de crise et à la communication opérationnelle (par exemple, la diffusion d'informations portant sur les activités au quotidien des membres de l'institution sur les théâtres d'opération, ce qui constitue sans doute une spécificité de ce ministère).

Le discours instituant que nous prenons ici en considération présente donc une première caractéristique essentielle : il vise à opérer le cadrage des discours émanant d'une institution. Discours portant sur ce que doit être le discours, il est constitué d'énoncés métadiscursifs.

Deuxièmement, et comme nous allons le voir, il s'agit d'un discours visant le « lissage » de la communication et des énoncés institutionnels en général et qui obéit lui-même à ce principe. Par lissage, nous entendons le gommage des formes de diversité et d'hétérogénéité en général.

Les formes de ce lissage peuvent être mises au jour par l'analyse de discours des textes de cadrage qui nous intéressent, alors même que des traces de tensions ou de polémiques continuent d'y affleurer. On voudrait mettre en évidence que c'est en croisant ces données (issues de l'analyse du discours instituant) avec l'analyse des discours produits par les acteurs de l'institution (lors de situations d'entretien avec le chercheur) que l'on peut avoir accès aux procédés de fabrication du discours instituant et, notamment, à des procédés de lissage. On pourra alors commencer à en dégager les enjeux communicationnels et politiques.

Pour résumer notre démarche, si l'analyse de discours approche le discours instituant comme produit, on peut accéder au processus de production lui-même par l'analyse des entretiens menés auprès des responsables de son élaboration ${ }^{2}$. L'idée de croisement ou de mise en résonance des deux approches est ici fondamentale puisque, selon nous, leur conjonction en une seule (anthropologie des discours institutionnels) est plus fertile que l'utilisation d'une seule d'entre elles. Les entretiens apportent à l'analyse de discours des données éclairant les conditions de production des discours analysés; l'analyse des discours instituants porte quant à elle sur les énoncés produits par cette activité, indépendamment du travail des chercheurs.

\section{Tensions à l'œuvre dans le discours instituant}

Les discours que nous étudions portent la marque de multiples tensions dont nous ne rappellerons que quelques formes ${ }^{3}$.

2. Ces entretiens portent alors principalement sur les méthodes de travail et amènent les acteurs institutionnels à en présenter au chercheur les enjeux et les objectifs, mais aussi à décrire le travail de production discursive.

3. Outre les références que nous ferons à nos travaux antérieurs, nous pouvons renvoyer à ce sujet à ceux de Marie-Anne Paveau, qui met par exemple en évidence, dans le discours militaire relatif 


\section{Traces indirectes de polémiques}

Le discours institutionnel porte la trace - souvent en partie gommée - de débats divers, qu'il s'agisse de polémiques internes, c'est-à-dire de dissensions plus ou moins clairement exprimées au sein de l'institution, ou de ce qui est perçu comme des attaques externes, c'est-à-dire de mises en cause réelles ou supposées de l'institution.

En ce qui concerne les polémiques internes, on peut ainsi repérer, dans les directives de communication de la Défense, la trace de tensions entre les tenants d'une communication "transparente », d'une "décrispation» des armées, d'une part, et la rémanence d'une méfiance, voire d'une hostilité envers les journalistes, d'autre part (Oger, 2003, p. 79).

Ces tensions, précisément en tant qu'elles apparaissent sous une forme affaiblie, sont en quelque sorte constitutives du discours officiel de la Défense en matière de communication en général et de relations avec les journalistes en particulier : elles ressortent en effet de l'analyse de documents institutionnels ainsi que de l'analyse d'entretiens avec les anciens responsables du SIRPA central ${ }^{4}$ (Ollivier-Yaniv, 2000). Elles prennent souvent une forme déniée ou sont mises à distance par des références au passé ou à une conjoncture exceptionnelle.

Plus généralement, le discours militaire envisage souvent l'activité des journalistes comme hostile et susceptible de rompre la cohésion du groupe (Oger, 2000), et cette menace continue à parcourir, y compris sous la forme de la dénégation, de nombreux textes.

De même, les récentes directives relatives au comportement du militaire ou à l'exercice de l'autorité font état de l'ensemble des dynamiques d'internationalisation comme d'autant de bouleversements qui remettent en cause l'identité militaire. Parmi ces dynamiques, la mondialisation de la communication (qui contraint notamment à compter avec les organes de presse étrangers) figure en bonne place, aux côtés de la construction européenne ou de l'internationalisation des opérations militaires sur certains théâtres (Oger, 2005).

Toutefois, ces tensions n'apparaissent généralement dans le discours institutionnel qu'à l'état de traces ou d'allusions et se trouvent recouvertes par un tissu serré de prescriptions et de normes.

aux relations avec les journalistes, l'existence de deux « scripts antithétiques » (Paveau, 2000). Dans une autre perspective, les recherches de G. Périès sur le discours militaire français pendant la guerre froide montrent comment le discours doctrinal procède à une étonnante captation et « re-torsion » du discours ennemi (Périès, 1999). Fabienne Pierre, enfin, observe les contradictions internes et les formes de neutralisation à l'œuvre dans le discours de l'ONU sur le « développement durable » ("Les discours institutionnels de la scène internationale. Cadres formels et informels du débat », communication à la Journée d'études CEDITEC / Paris 12-GRIPIC / CELSAParis 4, 21 janvier 2005).

4. Service d'information et de relations publiques des armées, remplacé en 1998 par la Délégation à l'information et à la communication de la défense (DICoD). 


\section{Prescriptions, formulations indirectes et contradictions}

À ces tensions externes, à ces risques de clivages internes, l'institution répond en effet par la production commune d'un discours qui apparait comme prescriptif à plusieurs niveaux.

Certains des textes que nous étudions appartiennent, comme nous l'avons dit, à la catégorie des directives, schémas directeurs, éléments de langage ${ }^{5}$ et, à ce titre, s'adressent à des destinataires « internes », c'est-à-dire à des acteurs institutionnels qui sont eux-mêmes producteurs de discours: leur activité se trouve ainsi encadrée par les normes et les principes édictés, ou même par le sens qu'il convient de donner aux mots ${ }^{6}$ ou encore par les mots qu'il convient d'utiliser (vocabulaire spécifique, syntagmes figés...). Ainsi la production du discours est-elle régie par un « système de contraintes » qui fixe les contours de l'énonciation légitime.

Dans un autre contexte, les campagnes de communication gouvernementale ont une dimension fondamentalement prescriptive puisque, s'adressant à l'ensemble des citoyens, elles leur enjoignent d'adopter un comportement particulier - notamment en matière de santé ou de sécurité, lorsque se trouvent énoncés les devoirs des citoyens.

Les campagnes de recrutement constituent à cet égard un cas particulier, puisqu'à la fois elles fixent une ligne de conduite interne (en énonçant les traits de l'identité légitime au sein de l'institution) et formulent une injonction externe (un récent slogan de l'armée de terre, «l'engagement par excellence», joue sur cette double dimension; en substance : « Notre métier est un véritable engagement... Engagez-vous»). Cette double injonction interne/externe pourrait se formuler comme suit : «Si vous êtes comme nous, rejoignez-nous, si vous nous rejoignez, soyez comme nous ».

Dans ces prescriptions, les contraintes sont évoquées de manière indirecte : ainsi la politique de «transparence » de l'institution militaire s'accompagne-telle d'un impératif - «parlez à votre niveau » - qui fonctionne concrètement comme une limitation drastique à l'expression des militaires. De même, l'impératif de cohérence (qu'on pourrait résumer par une injonction du type «parlons d'une seule voix ») comporte des prolongements et des conséquences qui en font un outil puissant de restriction de la parole.

L'activité de production de discours est ainsi soumise très officiellement et très explicitement (voir, par exemple, le décret de 1998 qui régit les activités de

5. Les éléments de langage sont des documents qui fournissent aux acteurs institutionnels des données (vocabulaire, chiffres, arguments) à intégrer à leur discours à l'attention de publics externes.

6. Ainsi le mot refondation [de l'armée de terre] peut-il recevoir sa définition officielle dans un argumentaire de 1999. 
la DICoD) à des injonctions contradictoires: effort de transparence et impératif de cohérence, respect de la vérité et souci de valoriser l'image de l'institution (Oger, 2003).

On trouve ce même mécanisme de contradiction dans les modalités de caractérisation du discours dit « citoyen » telles qu'elles apparaissent sur le site des forums gouvernementaux (www.forum.gouv.fr). Dans les pages de premier niveau du site, les forums sont présentés comme un « espace de dialogue » inscrivant la conduite de l'action gouvernementale «dans la transparence et la concertation »; dans les pages portant sur leur mode d'emploi, ils sont déclarés " ouverts à toutes et à tous ", constituant un "lieu d'échanges citoyens ». Par ailleurs, l'analyse de la charte d'utilisation met en évidence que la prise de parole obéit simultanément à des règles de modération, certes classiques mais parmi lesquelles, en plus des catégories de messages dont le rejet est juridiquement établi (propos promotionnels, racistes, diffamatoires, contraires aux droits d'auteurs...), on trouve les «messages non argumentés».

TOUTES les opinions qui respectent les points précédents ont leur place sur www. forum.gouv.fr. Toutefois, afin d'assurer une bonne qualité des échanges et des propositions, vous êtes invités à argumenter autant que possible vos contributions.

(Charte et mode d'utilisation des forums : forums.gouv.fr/infos.php3?id_article=5)

Le débat citoyen élargi, que le principe et la technologie du site forum sont censés favoriser, générer et encourager, se trouve donc simultanément contraint et limité par la restriction énoncée relativement aux qualités du discours et, ce faisant, par le caractère discriminant de la capacité des acteurs sociaux à prendre la parole.

Ces tensions et ces paradoxes, qui parcourent le discours instituant, c'està-dire la forme la plus officielle et la plus contrainte de discours institutionnel, peuvent être éclairés par l'analyse d'entretiens portant sur la fabrication du discours dans l'institution.

\section{Procédés de lissage discursif}

S'il ne subsiste, dans le discours officiel émanant de l'institution, que des traces de diversité ou d'hétérogénéité, de débats, de polémiques ou de difficultés, c'est en vertu d'un ensemble de procédés discursifs que nous qualifierons de «lissage».

Ce sont ces procédés qui donnent à lire ou à entendre un discours unifié et homogène, destiné au grand public (et non à des cercles plus ou moins larges d'initiés), dépourvu de formes individuelles de modalisation (en tant qu'il s'agit d'un phénomène d'énonciation collective) et placé à un haut niveau de généralité (en tant qu'il doit être valide dans de nombreuses circonstances). 
Nous nous référerons ici à des entretiens réalisés auprès de responsables et de membres de la DICoD par Claire Oger (Oger, 2003). D'autres entretiens mobilisés ici ont été réalisés par Caroline Ollivier-Yaniv auprès d'acteurs de la communication gouvernementale, qu'il s'agisse de membres du SIG ou de membres de cabinets ministériels ayant pour mission de préparer les prises de parole de ministres.

\section{Place du discours adverse et anticipation}

Les entretiens montrent que les communicants institutionnels consacrent une partie de leur activité à anticiper les questions qui pourraient leur être posées par des journalistes. À la Défense, cette activité prend notamment la forme d'une veille (à long terme : veille Internet sur des sites réputés hostiles à l'institution ; à plus court terme : veille « médiatique », c'est-à-dire télévision et quotidiens $^{8}$ ) ainsi que d'une activité collective de brainstorming pour anticiper les objections. En ce qui concerne la presse écrite et la télévision, cette activité se double a posteriori d'une évaluation de la réception du discours, par exemple dans des « analyses médias ».

De manière plus générale, il semble qu'une démarche d'analyse du discours médiatique (parfois à partir de méthodes importées du champ des sciences sociales) prenne progressivement le pas sur les traditionnelles revues de presse ou de médias, autrement dit sur la simple compilation d'énoncés journalistiques portant sur les activités de l'institution' ${ }^{9}$. Quand ils évoquent leur propre activité d'analyse, certains acteurs de la communication gouvernementale mettent en avant la plus-value d'information ainsi générée pour les responsables de l'institution: de leur discours émane alors le souci de faire connaitre les discours adverses ou concurrents aux porte-paroles de l'institution et, ce faisant, de leur permettre de les anticiper en vue de les neutraliser.

Tout se passe comme si la cohérence de la parole institutionnelle, lorsqu'elle est rendue publique, ne devait pas pouvoir être prise en défaut par une question imprévue.

Il faut noter que cette fonction qui repose sur la capacité à anticiper le discours adverse, y compris en formulant ouvertement les questions embarrassantes, semble le plus souvent confiée à des acteurs qui ne sont pas au contact direct des journalistes et qui restent quant à eux à l'abri de l'interaction.

7. Service d'information du gouvernement.

8. C'est le cas, en particulier, dans les entretiens menés par C. Oger en février 2003 auprès de l'ancien chef du département Stratégie ou auprès des porte-paroles de la DICoD.

9. C'est la mission de l'un des départements du Service d'information du gouvernement. 
L'opposition entre discours contradictoire-polémique et discours unifiécohérent apparait d'ailleurs dans les entretiens comme constitutive de la frontière intérieur/extérieur ${ }^{10}$ :

«Dans les argumentaires [...] toutes les questions sont posées [...], on ne cache rien [...], c'est un document qui reste interne, donc on n'a aucun intérêt à se priver de cette possibilité d'évoquer tous les sujets. Ce serait dommage [...]. Il faut tout aborder. Mais c'est après, dans la formulation de la réponse, où on fait attention à ne pas... à ne pas susciter d'autres questions, ou... à contourner la difficulté, tout en disant la vérité. [...] Je pense qu'en interne il faut l'avoir cette, cette liberté de ton. Et puis vers l'extérieur c'est autre chose, là il faut retrouver une cohérence dans ce que l’on dit. » (Ancien chef du département Stratégie, février 2003)

\section{Énonciation collective négociée}

Une partie du travail des communicants institutionnels consiste également à connaitre les éventuels débats, cette fois internes à l'institution qui n'en doit pas moins apparaitre comme un groupe cohérent et solidaire.

Ce point émane très clairement d'entretiens avec des écrivants de discours ministériels, dont l'activité consiste notamment à positionner la parole de son ministre relativement aux autres membres du gouvernement, au Premier ministre lui-même et, plus globalement, au discours gouvernemental - plutôt qu'à une pluralité de discours ministériels.

Abordant le positionnement, au sein du premier gouvernement Jospin, du ministre de l'Économie Dominique Strauss-Kahn, son écrivant évoque l'importance des relectures et de la réécriture des interviews accordés par le ministre pour obtenir un discours qu'il qualifie dans un premier temps de "politiquement correct» puis de « jospino-compatible» (lorsqu'une relance le conduit à préciser).

Par l'analyse du discours d'un écrivant de Dominique Voynet, on comprend également que l'institutionnalisation d'un discours ministériel et du ministre qui le porte (même lorsque la personne considérée est également chef de parti politique) passe par le fait de mieux circonscrire les sujets sur lesquels on s'exprime et par la réduction des conflits rendus publics. Cet écrivant, observant en effet que les discours de la ministre en dehors de son champ de compétences se sont raréfiés après dix-huit mois de présence au gouvernement, commente :

10. De même, lorsque la cohérence du discours et la cohésion du groupe sont remises en question, la production du discours institutionnel tend à colmater les brèches ainsi produites en réaffirmant les fondements de l'identité commune (Oger, 2000 ; Oger, 2005). Il s'agit alors de prévenir les « dérives » possibles et, là aussi, de ramener la diversité et l'hétérogénéité à l'unité d'un comportement et d'un discours communs. 
Et la deuxième période, malheureusement ou heureusement, je n'en sais rien, toujours est-il que... peut-être par lassitude, par fatigue, par manque de temps... L'impression que j'ai eue, c'est qu'elle est beaucoup moins venue sur les autres sujets. Elle est restée en retrait. Elle est restée... Elle est devenue, sans doute, une ministre plus classique... à s'exprimer sur son seul périmètre. C'est aussi que Jospin, quand elle a compris qu'il ne lâcherait pas... D'abord, il l'a remise en place. Mais même qu'il ne lâcherait rien sur les sans-papiers, qu'il lâcherait rien sur euh... C'était tactique, oui. Pour des raisons tactiques mais plutôt stratégiques je pense qu'elle a rapidement fait l'analyse - ou c'était intuitif - qu'elle ferait plus avancer les choses vite, et donc c'est une affaire politique, dans une posture de négociation avec Jospin, permanente, plutôt que dans une posture de, de... de radicalisation, de positions franches. (Entretien du 21 juillet 2003)

Dans les entretiens menés avec des membres de la DICoD, une pratique similaire consiste à mettre à jour les déclarations en y réinjectant des éléments suggérés soit par les services des autres ministères, soit par le cabinet : là encore, l'objectif est l'harmonisation des discours :

Le deuxième travail c'est donc, à partir de cette matière première, il y a un certain nombre de... qui, qui, encore une fois peut dater de, des, des, des différentes périodes, il faut l'actualiser, [...] alors en fonction de deux éléments, donc, je dirais... qui sont les inputs : d'une part les directives... politiques, donc euh, du cabinet [...] voire du cabinet, enfin, de la présidence de la République, et/ou [...] des Affaires étrangères, donc y a des directives politiques qui nous viennent [...] la plupart du temps par l'intermédiaire [...] du porte-parole, donc de Monsieur Jean-François Bureau. Et par ailleurs, deuxième input, c'est que nous avons des, des liens je dirais transversaux avec entre guillemets nos homologues [...], qui sont d'une part les services du porte-parole des Affaires étrangères, [...] le service d'information du gouvernement, principalement, les correspondants... Je dirais qui font le même travail que nous auprès du ministre de l'Intérieur et au cabinet du Premier ministre. Bon, et donc notre rôle, c'est effectivement [...] de sortir [...] un discours qui soit harmonisé au niveau du gouvernement, et donc nous-mêmes, nous, entre guillemets, nous "lissons », nous travaillons, à partir des éléments qui nous sont donnés, par ces différents correspondants..., nous lissons la parole. (DICoD, Bureau porte-parole, février 2003)

Lorsque des positions et des discours concurrents existent au sein de l'institution, un autre procédé discursif de lissage du discours institutionnel procède donc d'une énonciation collective négociée. Selon cette double perspective d'intégration des discours concurrents extérieurs et internes à l'institution, la production du discours institutionnel s'accompagne donc d'un effort de réduction de l'hétérogène à l'homogène.

\section{Montée en généralité et décontextualisation}

Une autre dimension du « lissage » du discours perceptible dans les entretiens est celui de sa « décontextualisation ». 
Évoquant la fabrication des " éléments de langage " $^{11}$, les responsables du bureau porte-parole de la DICoD évoquent deux formes de «lissage ». La première consiste à détacher la déclaration officielle (déclaration d'une autorité reprise dans les éléments de langage) de son contexte d'énonciation particulier pour lui donner une valeur plus générale et une intelligibilité indépendante du contexte ${ }^{12}$ :

C'est l'ensemble des... déclarations, interviews..., rencontres, etc., que, que les différentes autorités habilitées, [...] président de la République, ministre des Affaires étrangères, ministère de la Défense bien évidemment, chef d'état-major des armées éventuellement, donc l'ensemble des interventions que ces gens-là ont faites dans le temps, et devant des auditoires particuliers, donc..., c'est surtout ça qui est important, c'est qu'on travaille à partir d'une parole qui a été prononcée devant euh..., des anciens combattants y a deux mois, puis devant des parlementaires de l'Union... de l'Europe Occidentale y a... y a quinze jours, et puis devant, euh, des chercheurs [...], y a trois jours. D'accord? Et le public, donc, auquel on s'adresse, conditionne la façon dont on parle, et donc il s'agit, euh, de, de transformer cette parole officielle, de, de la malaxer si j'puis dire, pour la rendre intelligente, pertinente et compréhensible par les médias. Ça c'est le premier travail. (DICoD, Bureau porte-parole, février 2003)

Une autre forme de lissage consiste, en cas d'alternance ou de remaniement ministériel, à gommer les noms propres des ministres pour ne laisser subsister que la mention impersonnelle de leur fonction :

Parce que maintenant, on peut dire qu'y a un nouveau gouvernement, $y$ a des nouvelles déclarations, donc quand les gens nous demandent par exemple, euh..., quelque chose sur l'ART, évidemment, on lisse les éléments de langage précédent, c'est-à-dire qu'on enlève le nom et le prénom du ministre, on met «le ministre de la Défense ». (DICoD, Bureau porte-parole, février 2003)

Dans ces passages, lissage et mise à jour vont de pair. On peut ainsi considérer que le discours instituant visé est un discours :

- qui peut être porté par tout énonciateur institutionnel;

11. Comme les argumentaires, les éléments de langage sont fabriqués sous la forme de questionsréponses, proposant à des questions « de type journalistique » des réponses-types qui formulent la position officielle de l'institution : «La seule façon qu'on a trouvée pour l'instant, c'est de le rédiger sous la forme de questions-réponses, [...] questions en fait..., type journaliste, c'està-dire qu'on n'essaie pas de faire des questions type langue de bois, c'est-à-dire que c'est des questions qui, qui peuvent être posées par des journalistes, et on y répond, comme si on..., dans le format, je dirais, de la réponse d'une minute trente, devant une caméra, ou même de trente secondes. Donc on essaye de se calquer sur... la dynamique vivante du..., du contact... presse. » (entretien Bureau porte-parole de la DICoD, février 2003). À la différence des argumentaires qui anticipent à plus long terme, les éléments de langage s'appuient généralement sur le discours déjà produit par les autorités sur le sujet.

12. On reconnait là un procédé très proche de celui qui est décrit par Dominique Maingueneau lorsqu'il évoque l'existence et la transformation des «énoncés détachables» (Maingueneau, 2005). 
- qui peut être reçu facilement (les adjectifs « compréhensible », « clair »... sont récurrents) par le public le plus large : les journalistes et, au-delà, le grand public ;

- qui est détaché des circonstances particulières de son énonciation (lorsqu'il est, comme dans les éléments de langage, repris du discours officiel des autorités) ;

- qui est mis à jour en permanence et ainsi toujours « actuel » et prêt à être prononcé.

\section{Gommage du politique}

Plusieurs caractéristiques évoquées précédemment contribuent à un gommage (pour ne pas dire à un déni) de la dimension politique de l'institution, gommage identifiable sous deux procédés: le changement de temporalité et l'effacement de la politique partisane.

La décontextualisation des énoncés rend ainsi une déclaration intelligible en dehors et finalement indépendamment de son contexte d'énonciation et rompt la dépendance entre les énoncés et leurs énonciateurs originels, ou entre les énoncés et les destinataires originels. Ce procédé tend à imprimer au discours une temporalité sur le long terme, pour ne pas dire un caractère universel. Cette temporalité est bien caractéristique de l'institution et non de ses représentants et membres successifs et temporaires.

Ces échelles de temps distinctes sont d'ailleurs souvent présentées comme étant décalées par les spécialistes de la communication gouvernementale institutionnelle : le temps du politique apparait le plus souvent comme un facteur de perturbation, en particulier parce qu'il altère les conditions de réussite et d'efficacité des messages destinés aux citoyens. Au moment de leur mise en œuvre, bon nombre de campagnes de communication gouvernementales ont en effet vocation - explicite - à "s’inscrire dans la durée », ainsi que le mentionne le dossier de presse de la campagne 2000 de lutte contre la drogue et de prévention des dépendances, dont les postulats furent pourtant très discutés dans l'espace public ${ }^{13}$.

De même, Jean-François Bureau insiste sur sa fonction de porte-parole du ministère de la Défense (et non du ministre) et explique par cette position institutionnelle sa longévité dans le poste, peu soumis selon lui aux aléas de l'alternance politique.

Il existe également des procédés tangibles d'effacement du politique en matière de campagnes de communication gouvernementale.

Ainsi, l'un des principes fondateurs de ces campagnes consiste à ne pas faire apparaitre le ministre qui en porte la responsabilité. Il en est ainsi pour les

13. «Drogues : Savoir plus, risquer moins », campagne lancée le 26 avril 2000. 
campagnes qui visent «à promouvoir des modifications souhaitables de comportement du public dans certains domaines de la vie collective, en matière de santé, de sécurité routière, de protection de l'environnement par exemple", mais aussi pour les campagnes qui ont « pour objectif de porter à la connaissance du public les mesures ou les dispositifs nouveaux arrêtés pour mettre en œuvre la politique du gouvernement $»^{14}$.

Les arguments développés pour justifier sont majoritairement d'ordre professionnel et renvoient alors à une expertise en matière de communication : le fait de faire figurer un(e) ministre n'est pas cohérent avec l'objectif de construire un message potentiellement efficace - car en adéquation avec les cibles visées -, la présence du représentant du ministère étant alors perçue comme une source de brouillage.

À bien des égards, si l'on se réfère tant aux documents de cadrage qu'aux entretiens avec les chargés de communication, le discours des campagnes de communication gouvernementales est donc construit comme si leur mise en œuvre ne procédait pas de choix politiques, alors même que certaines campagnes procèdent de décisions circonstanciées. Il arrive en effet que les ministres s'impliquent dans la présentation de campagnes de communication ou que certaines campagnes de communication institutionnelles ne soient pas reconduites ou prolongées en vertu des choix de la politique gouvernementale. Il en est par exemple ainsi des campagnes d'information sur la contraception, dont l'importance avait été affirmée en 1999, notamment par plusieurs déclarations de la ministre de l'époque Martine Aubry, et dont la périodicité était censée être bisannuelle. Si elle a en effet été reconduite en $2002^{15}$, elle n'a pas eu de suite et aucune campagne de communication en matière de contraception n'était à l'époque sur l'agenda du ministère de la Santé du gouvernement Raffarin.

\section{Sens et enjeux}

Nous avons donc été ici amenées à mettre en résonance plusieurs niveaux de discours institutionnels imbriqués dans la sphère ministérielle et gouvernementale et dont il est difficile de spécifier la place exacte dans un continuum qui va du discours de l'institution au discours politique :

- métadiscours portant plus particulièrement sur le cadrage de la communication des institutions;

- campagnes de communication institutionnelle (présentées comme autonomes de la sphère politique);

14. Guide des campagnes d'information gouvernementales, brochure diffusée par le Service d'information du gouvernement en 1996, p. 3.

15. « Le gouvernement s'est engagé depuis deux ans à mener des campagnes d'information régulières sur la contraception afin de permettre aux femmes de mieux maitriser leur fécondité et ainsi prévenir les IVG. » (Dossier de presse 2002) 
- discours du porte-parole de l'institution (ministères des Affaires étrangères et de la Défense) ;

- discours des représentants de l'institution (ministres, secrétaires d'État) ;

- discours (suscités par entretien) des acteurs de l'institution qui produisent ceux qui précèdent.

\section{Sens communicationnel}

De ces différentes analyses se dégage la conception fonctionnelle de la communication qui sous-tend la production du discours dans les institutions.

La démarche décrite vise globalement à réduire les sources de bruit et recherche l'efficacité. L'adaptation à l'auditoire (notamment par l'identification de publics-cibles - journalistes, grand public ou certaines de ses catégories) est bien énoncée comme un objectif. La conception du discours (ou du message) est déterminée par un souci de clarté : on utilise l'explication, parfois l'injonction, plus rarement l'argumentation.

En revanche, les procédés de lissage peuvent être définis comme des procédés de rationalisation du débat en particulier et du discours en général. Tout se passe donc comme si on ne doutait pas de la solution proposée mais de son intelligibilité ${ }^{16}$. On vise un discours qui aurait un statut de vérité, vérité dont l'institution se trouve par là même détentrice.

\section{Enjeux politiques}

Ce « discours de vérité » se construit simultanément comme discours d'autorité fonctionnant sur le mode de la normalisation plutôt que sur celui de la contrainte objective.

Trois mécanismes semblent identifiables au travers des procédés de lissage du discours instituant observés précédemment : neutraliser le débat, soumettre le discours des acteurs à des injonctions contradictoires, construire une idéologie de l'intérêt général et de l'action publique.

L'objectif de neutralisation des discours concurrents ne passe pas par une démarche contraignante et encore moins une censure. Il repose sur des savoirfaire qui permettent leur mise au jour et leur anticipation, autrement dit sur des modalités de contrôle et d'ajustement de discours concurrents, parfois contradictoires.

16. Voir Bronckart, 1996: «On peut considérer que lorsque l'agent producteur estime qu'un objet de discours, pourtant incontestable à ses yeux, risque d'être problématique (difficile à comprendre) pour le destinataire, il tend à développer la présentation des propriétés de cet objet en une séquence explicative. Et lorsque l'agent producteur estime qu'un aspect du thème qu'il expose est contestable (à ses yeux et/ou à ceux du destinataire), il tend à organiser cet objet de discours selon une séquence argumentative. » (Bronckart, 1996, p. 237, souligné par l'auteur) 
On peut poser l'hypothèse que c'est ce type de démarche qui est également à l'œuvre lorsqu'une institution se définit des «partenaires» (associatifs, par exemple) en vue de constituer un message « négocié » ou de mieux peser sur le message que ces acteurs feront sortir de l'institution.

Il en est encore ainsi du travail des communicants de la Défense pour la préparation du point presse hebdomadaire du délégué à la communication : en anticipant les questions des journalistes, on vise à la fois à déjouer des mises en cause de l'institution et à produire un discours qui est susceptible d'être relayé par les journalistes.

La neutralisation du débat va donc ici de pair avec la rationalisation et la maitrise du discours.

Parallèlement, le discours des acteurs sociaux - ceux qui sont à l'intérieur de l'institution et ceux qui s'adressent à elle - se trouve ainsi soumis à des contraintes contradictoires.

L'institution militaire doit être «transparente» mais chacun doit «parler à son propre niveau ». L'action gouvernementale doit être conduite «dans la transparence et la concertation », notamment grâce aux forums qui sont des lieux "d'échanges citoyens » ouverts à tous, mais surtout à ceux qui savent argumenter correctement.

Le caractère paradoxal du discours instituant qui décrète la communication et ses bienfaits est enfin perceptible lorsqu'on analyse le discours et les activités de certains de ceux qui sont censés œuvrer à la bonne diffusion du discours et de la communication institutionnelle et dont on considère qu'ils ont précisément vocation à demeurer dans l'ombre. Il en est ainsi des membres du SIG, communicants gouvernementaux institutionnels ou encore des écrivants, seuls membres des cabinets ministériels dont on préfère ne pas ébruiter l'existence aux yeux du grand public. C'est moins le contenu dissimulé - puisqu'il s'agit d'un secret de Polichinelle - que le sens de cette occultation qui est intéressant (Ollivier-Yaniv, 2003b).

Enfin, comme on l'a évoqué précédemment, le discours instituant est positionné, de manière discursive, au-dessus des représentants temporaires de l'institution et comme indépendamment de la temporalité politique. Le discours instituant se donne donc à entendre comme s'il était le reflet de l'intérêt général, alors qu'il participe en fait à sa construction à un moment donné.

Quant à la communication des institutions gouvernementales (dite « communication publique»), elle définit et elle édicte par le discours (à caractère fondamentalement prescriptif et injonctif) les droits des citoyens en étant justifiée par la recherche du bien commun et en étant fondée sur la responsabilisation des individus relativement à la société. Ce faisant, elle fonctionne comme complément ou comme substitut à des procédés de coercition : complément, par exemple, dans le cas de la sécurité routière, substitut, par exemple, relativement aux thématiques de santé publique.

Considérant les procédés de lissage du discours instituant, on est conduit à 
dessiner les contours d'un « discours de vérité » qui a valeur (pour ne pas dire fait office) de discours d'autorité.

Dans cette perspective, le lissage à l'œuvre dans le discours officiel et dans la communication des institutions gouvernementales constitue une forme de contrainte symbolique dont on peut penser qu'elle élabore un véritable outil de gouvernement dans un cadre démocratique.

En d'autres termes, discours instituant et communication institutionnelle n’invalident pas un certain nombre de principes démocratiques - liberté et égalité dans la prise de parole, nécessité du débat public -, dont ils vont même jusqu'à se réclamer.

Placé sous l'autorité du Premier ministre, le SIG favorise les échanges entre les citoyens et le gouvernement. [...] Le droit à l'information sur l'action du gouvernement est une exigence de la démocratie ${ }^{17}$.

Ils n'en fixent pas moins les cadres et les normes des discours et des comportements, œuvrant ainsi comme des outils de régulation de l'espace public.

\section{Références}

BRONCKART J.-P., 1996, Activité langagière, textes et discours. Pour un interactionnisme socio-discursif, Lausanne, Delachaux et Niestlé.

MAINGUENEAU D., 2005, «De la surassertion à l'aphorisation », Actes du $2^{\mathrm{e}}$ Colloque international du groupe Ci-Dit, Cadiz, 11-14 mars 2004.

OGER C., 2000, « De l'esprit de corps au corps du texte : cohésion militaire et dissolution journalistique », Langage et société, $n^{\circ}$ 94, décembre, p. 9-43

2003 : «Communication et contrôle de la parole. De la clôture à la mise en scène de l'institution militaire », Quaderni, ${ }^{\circ}{ }^{\circ}$ 2, automne, p. 77-92.

2004: "Des frontières nationales aux frontières institutionnelles. Mémoire discursive et identité de l'armée de terre ", Les Champs de Mars, $\mathrm{n}^{\circ} 16,2^{\mathrm{e}}$ semestre, p. $159-184$.

et OLLIVIER-YANIVC., 2003a, "Conjuguer analyse du discours institutionnel et sociologie compréhensive. Vers une anthropologie des discours institutionnels», Mots. Les langages du politique, $\mathrm{n}^{\circ} 71$, mars, p. 125-145.

2003b: «Du discours de l'institution aux discours institutionnels. Vers la constitution de corpus hétérogènes ", $1^{\text {re }}$ Conférence internationale francophone en sciences de l'information et de la communication (CIFSIC), Bucarest, 28 juin-2 juillet 2003. Texte disponible sur archivesic.ccsd.cnrs.

OLLIVIER-YANIV C., 2000, "Quels “professionnels” pour la communication et les relations avec les médias à la Défense ? Carrière militaire et communication », Langage et Société, $\mathrm{n}^{\circ}$ 94, décembre, p. 75-96.

17. Texte de définition du SIG sur le site du Premier ministre, 1997, http://www.archives. premierministre.gouv.fr/jospin, 17 janvier 2005. 
_ 2003a, "La fabrique du discours politique. Les «écrivants » des prises de parole ministérielles», Rhétorique et discours politique, Actes du Colloque de Cerisy, Rennes, Presses universitaires de Rennes, p. 89-98.

- 2003b, «Les communicants gouvernementaux au secret: croire et faire croire à la transparence du politique », Quaderni, $\mathrm{n}^{\circ}$ 52, automne 2003, p. 105-116.

PAVEAU M.-A., 2000 : "Les frontières discursives de la militarité », Langage et société, $\mathrm{n}^{\circ} 94$, p. 45-74.

PÉRIĖS G., 1999, "L'appel à l'ennemi, structure de la création doctrinale pendant la guerre froide », Thiéblemont A. éd., Cultures et logiques militaires, Paris, PUF, p. 289339.

RANGEON F., 1986, L'idéologie de l'intérêt général, Paris, Economica. 